\title{
Del contenido al sentido: estereotipos de la vejez como prácticas discursivas
}

Miguel Ángel Sahagún Padilla, ${ }^{1}$ Pedro Palacios Salas, ${ }^{1}$ Anna Vitores González, ${ }^{2}$ Cecilia Méndez Sánchez, ${ }^{1}$ Alicia Edith Hermosillo de la Torre ${ }^{1}$ y Francisco J. Elejabarrieta Olabarri ${ }^{3}$

\section{Introducción}

El concepto de estereotipo es uno de los más emblemáticos de la psicología social, aunque se encuentra muy lejos de serlo en condiciones de exclusividad. Tal es la amplitud de uso del concepto -con todas las objeciones de definición que se le quieran poner- que no resulta extraño encontrarlo incorporado a oraciones producidas en el contexto de conversaciones cotidianas en los más diversos escenarios. Desde Lippmann hasta Fiske y desde la situación institucional más tensa hasta la escena de sitcom más relajada, la idea de estereotipo como generalización exagerada se mantiene a flote. Eso sí, con las más diversas acotaciones, matices y valoraciones. Si de exageraciones se trata, uno podría incluso llegar a decir que la idea de estereotipo está estereotipada.

1 Universidad Autónoma de Aguascalientes.

2 Lancaster University.

3 Universitat Autònoma de Barcelona. 
No podemos ignorar que hay discusiones claramente establecidas sobre el lugar de los estereotipos en las esferas psicológica y social de la existencia, como por ejemplo la relativa a si sus efectos son necesariamente perniciosos o la que tiene que ver con que sean o no inevitables o inherentes al procesamiento de información. Pero, en discusiones como las anteriores, la concepción de estereotipo como creencia funcionaría como constante, como telón de fondo genérico y generalizado al que nadie tiene que prestar atención precisamente porque todos saben que está ahí y que funciona como base para el debate. Se asume, pues, el estereotipo como conjunto de rasgos asociados a determinada categoría social. Ese conjunto de rasgos -siempre según nuestro discreto telón de fondo- puede movilizarse de diferentes maneras; es decir, se expresa de formas distintas y tiene, por tanto, distintas funciones o usos. Desde esta concepción, una cosa es el estereotipo y otra su expresión o uso. Distinguir entre ambos no sólo es posible sino imprescindible.

El trabajo que presentamos aquí no pretende deslindarse por completo de esta pauta bien establecida en el tratamiento de los estereotipos. Nos interesa, eso sí, desdibujar la distinción, tan obvia en apariencia, entre el estereotipo y las formas en que se expresa o las funciones que la expresión cumple. Nos interesa, para decirlo de otro modo, proponer una alternativa a la distinción entre pensamiento y práctica en el caso específico de los estereotipos. Para ello, presentaremos un esbozo de aproximación conceptual a los estereotipos a partir de una orientación praxeológico-discursiva. Específicamente, hablaremos de la centralidad de la desigualdad que promueven los estereotipos, del carácter relativo en la valencia de los rasgos que los componen y, por último, de sus especificidades discursiva, demográfica y cotidiana. Posteriormente, señalaremos algunas implicaciones metodológicas de la aproximación descrita y algunas propuestas para su necesario desarrollo. Llevar a los estereotipos del contenido al sentido implicaría arribar al terreno del significado desde una orientación pragmatista, atenta a los usos y escenarios como elementos indisolubles. 


\section{Desarrollo}

El esquema conceptual que presentamos a continuación no pretende ser un producto final, exportable en cualquier circunstancia. Tampoco un punto y aparte en el estudio de la discriminación. Nos interesa presentar con un mínimo de claridad las pautas desde las cuales estamos ensayando una forma de entender los estereotipos en la investigación. Nos interesa esclarecer nuestro proceder para poder comunicarlo. Nos interesa, en suma, hacer un esfuerzo de inteligibilidad de modo que podamos participar con sentido en esa conversación amplia, larga y diversificada sobre nuestras formas de caracterizar al otro y de relacionarnos con él.

\section{Desigualdad social}

El primer punto que nos gustaría abordar es el de la centralidad de la desigualdad social en la comprensión de los estereotipos. La aproximación sociocognitiva a los estereotipos es, sin duda, la más ampliamente utilizada en la actualidad, al menos en el ámbito de la psicología social. La idea central de esta aproximación es la inevitabilidad de recurrir a estereotipos. Traduciendo a Fiske y Dupree (en prensa: 3), "juzgar a otras personas por su pertenencia a una categoría social amplia, sea étnica, de género, de edad o de clase social, es un atajo común que todo el mundo utiliza, para bien o para mal". Los estereotipos serían el producto de, si se nos permite la expresión, unos "atajos cognitivos" sin los cuales sería imposible funcionar. La labor de una psicología cognitiva cada vez más tendiente a la neurociencia sería iluminar los procesos de generación y uso de los estereotipos; procesos de naturaleza individual que en términos de contenido se nutrirían de su medio cultural. En línea con las observaciones de Agoustinos y Walker (1998), una aproximación de esta índole descuida considerablemente las complejas tramas de relaciones de poder que conforman el mundo social en el que los estereotipos se producen y reproducen; las tensiones intergrupales en las que tienen sentido a partir de las funciones ideológicas en las que participan. 
Ésta es, desde nuestro punto de vista, una debilidad mayor de la perspectiva sociocognitiva sobre estereotipos, ya que en términos prácticos elimina de la explicación un aspecto central del estereotipo o le da un lugar más bien periférico. En otras palabras, si somos sensibles a la historia del concepto y a las funciones que, se supone, desempeñaría en el marco de la producción y aplicación de conocimiento psicosocial, los estereotipos serían reconocibles no sólo como resultado de unos procesos cognitivos genéricos, sino como productos con determinadas descripciones (contenidos que responden a relaciones desiguales entre categorías sociales) y, simultáneamente, determinadas prescripciones (formas de legitimación, funciones ideológicas). Los alcances de las formas de dar cuenta de la durabilidad de un estereotipo, de su dureza, atendiendo a la etimología de la palabra, dependerán en buena de medida de si consideran o no esas tramas de poder entre categorías sociales en las que el estereotipo tiene su sentido y funciones. Así, un estereotipo se caracteriza y delimita en función de las relaciones entre categorías sociales que está mediando, específicamente por la forma de desigualdad que promueve. No es tanto -o por lo menos esto no es lo relevante- una modalidad fundamental de procesamiento de la información como un juego de adscripción de atributos basado en la categorización que promueve relaciones de poder mediante la enunciación de diferencias con valor entre tales categorías sociales.

\section{Relatividad del rasgo}

El segundo punto es el del carácter relativo y contextual de los rasgos o atributos que integran los estereotipos. Hacer referencia al carácter relativo de los rasgos implica distanciarse de los supuestos de linealidad y atomicidad. La atomicidad iguala estereotipo con atributo; entiende el estereotipo como rasgo independiente adscrito a la categoría social. El estereotipo es el rasgo identificado con la categoría y que, por extensión, se aplica a los objetos contenidos en la categoría. La linealidad supone que la valencia del rasgo aislado y descontextualizado coincide con la valencia del estereotipo. 
Hablar de la relatividad de los estereotipos significa preguntarse por la valencia no en función del rasgo en sí, sino desde la relación jerárquica del rasgo con otros rasgos y esto, evidentemente, sólo tiene sentido en determinados espacios de la vida social. Dicho de otro modo, la valencia, el carácter positivo o negativo de la atribución, se deriva de la posición del atributo en la escala de atributos relevantes en un campo social dado. Así, hablar de candor y calidez en un espacio social de alta competitividad como la bolsa probablemente no remita a un atributo deseable o altamente valorado.

Para añadir algo más de complejidad a la cuestión, tenemos el problema, también relacionado con la relatividad, de que el conjunto de atributos aglutinados en torno a la categoría es producto de una selección coherente e interesada -que no deliberada o consciente- que considerará determinados atributos e ignorará otros, justamente porque, para que los conjuntos de adscripciones tengan sentido, es preciso mantener fuera del conjunto -ignorar- esos otros rasgos. Finalmente, habría que recordar que, desde la aproximación que defendemos, identificar o delimitar estereotipos implica algo más que objetivar o reconstruir esos sistemas de rasgos relacionados en el marco de determinados campos; también implica dar cuenta de su uso. Se trata de un uso que, volviéndonos a distanciar del esquema "estereotipo = creencias $\rightarrow$ discriminación = acción", dista mucho de ser una expresión lineal y estándar y que se desarrolla de múltiples formas, integrando no sólo instancias de categorización, sino además de particularización (Billig, 1996) y considerando el papel que juega en esas prácticas la noción de prejuicio que los participantes puedan tener (Billig, 1991). Así, para hablar de estereotipos, necesariamente habría que integrar en un mismo plano el de la creencia o representación y el del comportamiento o práctica.

\section{Especificidad discursiva}

El tercer punto remite a la especificidad discursiva de los estereotipos. Se trata de una pregunta inexcusable. Las diversas modalidades de análisis del discurso en psicología social se pro- 
ponen justamente como una alternativa sostenible a los clásicos estudios de estereotipos y actitudes (Íñiguez, 2003; Potter y Wetherell, 1987; McKinlay y McVittie, 2008). Si esto es así, cabe entonces preguntarse por la necesidad de seguir hablando de estereotipos cuando bien podría hablarse de discurso, en alguna de las aproximaciones existentes. Preservar la noción de estereotipo desde la transformación del propio concepto posibilitaría, entre otras cosas, la consideración de buena parte de los estudios sobre el tema (e.g. estudios correlacionales tipo encuesta), reinterpretándolos en otro plano. Pero estas razones sostienen la pertinencia de la aproximación, no su factibilidad.

En cuanto a la especificidad discursiva, que daría factibilidad a esa pertinencia, se la puede encontrar tanto en la función, que remite a la consideración de un objeto, en este caso persona mayor, en unos términos que le reducen a un conjunto de propiedades -el significado- atribuidas a una categoría social, como en la forma, caracterizada por el uso de expresiones hechas o lugares comunes, con mayores o menores modificaciones y con mayor o menor fortuna para el interlocutor, considerando la situación o escenario en que se produce la enunciación (característica que también aplica a textos y otros formatos). La impertinencia, discutibilidad o impunidad retórica con la que pueda practicarse el lugar común serían un indicador del valor a la alza o a la baja del estereotipo en determinados ámbitos de la vida social y, para complicar aún más las cosas, daría también seña de su temporalidad. La unidad función-significado, base para la inteligibilidad del estereotipo, nos recuerda que contenido y significado no son la misma cosa; nos recuerda, en suma, que el significado del estereotipo es inherente a la práctica; a lo que hacen determinados contenidos expresados de determinadas formas en determinados contextos. El contenido de un estereotipo es fácilmente evidenciable; siempre se pueden presentar argumentos -algunos extremadamente sencillos- para desmontar la sobregeneralización. Es la práctica, el hábito, lo que no resulta fácil de desmontar. La dureza del estereotipo es entonces la dureza de un hábito de categorización social. 


\section{Especificidades demográfica y cotidiana}

Hasta ahora, lo que hemos señalado aplica a la noción de estereotipos en general. Ahora nos interesa hacer un pequeño alto para visitar algunas especificidades propias de los estereotipos hacia la gente mayor. Especificidades como éstas ayudan a establecer el horizonte de interrogación que debería definir un estudio sobre la materia desde la perspectiva arriba esbozada.

El horizonte común de todo ser humano es, normalmente, llegar a ser una persona mayor. Retóricamente, esto supone que los argumentos que recurren a expectativas y prospectivas en primera persona son no sólo viables sino muy proclives a tener lugar. De manera semejante, toda población cuenta con cierto porcentaje de adultos mayores, con la contrapartida más próxima de que casi todo individuo se ha relacionado con adultos mayores en el seno familiar y prácticamente todo individuo, con mayor o menor distancia, en el marco de su vida cotidiana. O la insistente preocupación con la que, en las más diversas sociedades, se habla de la inversión de la pirámide poblacional y de la necesidad de prever formas de sostener la calidad de vida de la tercera edad, que se acompaña de consignas altamente normativas como la de tener una vejez "exitosa" o "activa".

\section{Conclusiones}

Sintetizando las consideraciones expuestas, un estereotipo remite a un conjunto de prácticas lingüísticas que reproducen un concepto dado sobre una categoría social cuyos rasgos implican una relación asimétrica desfavorable para dicha categoría social en relación con otra u otras categorías en determinado campo de la vida social. Desde esta concepción, se rescata lo específico de los estereotipos de una forma crítica y situada, útil cuando lo que se pretende es configurar una aproximación psicosocial a la reproducción de la desigualdad.

Las implicaciones para la práctica del análisis son varias. Algunas tienen la forma de prescripción que habría que seguir, 
mientras otras tienen la forma de pregunta que habrá que responder para concretar un abordaje empírico al fenómeno. En primer lugar, está la cuestión del dato, la pregunta por el referente empírico pertinente para dar cuenta de los estereotipos hacia la gente mayor. Desde la perspectiva presentada resultará evidente que una aproximación naturalista a los entornos de actividad y relación relevantes para la gente mayor sería lo deseable. Esto no significa, sin embargo, que otros métodos no puedan hacer aportaciones específicas, siempre que éstas sean congruentes con las preguntas que pretende responder un estudio y se tenga claro cómo tratar la información desde el marco descrito. Una encuesta bien diseñada, por ejemplo, podría ser útil para identificar tendencias en la reconfiguración o permanencia de los contenidos de determinados estereotipos o para conocer cómo su mayor o menor discutibilidad se distribuiría en una población. En la misma línea, una entrevista semiestructurada, abordada en términos de conversación, constituiría un espacio de despliegue de estereotipos en tanto que prácticas. Los roles desde los cuales se incluiría a los participantes y los efectos que tendrían sus antecedentes serían tratables desde la propia lógica discursiva, desde la forma en que se materializan y hacen presentes en el propio discurso.

En cuanto a análisis, se podrían considerar conjuntos parciales de los aspectos que aparecen a continuación, en función de los objetivos del trabajo por realizar. Aspectos como: a) los rasgos o propiedades adscritos a la categoría y su carácter de atribución generalizada; b) los sistemas o, al menos, los conjuntos que conformarían; c) las características o modalidades de las expresiones específicas que sostienen las adscripciones, particularmente aquellas que remiten a la experiencia, proximidad o inexorabilidad de la vejez; d) la familiaridad con cada expresión, el grado en que se aproxima a, o constituye un lugar común y su mayor o menor sentido de expresión políticamente correcta; e) f) los rasgos opuestos -tácita o explícitamente- a los rasgos adscritos a la categoría y los pares que conforman; g) la desigualdad entre categorías sociales y el tipo de relación social promovidas por la categorización, así como su participación en la reproducción del 
orden social; h) la presencia y la relación de formas de particularización con la categorización; i) nociones de estereotipo, prejuicio, discriminación, cliché u otras semejantes usadas por parte de los actores involucrados en la práctica y el tipo de uso (e.g., la acusación de usar estereotipos) y, finalmente, j) el marco social en el que se despliega la práctica, el campo específico en el que su inteligibilidad sólo es posible en cierta dirección.

\section{Referencias}

Augoustinos, M. y Walker, I. (1998). The Construction of Stereotypes within Social Psychology: From Social Cognition to Ideology Theory. Theory \& Psychology 8(5), 629-652.

Billig, M. (1996). Arguing and Thinking: A Rhetorical Approach to Social Psychology (2 ed). Cambridge: Cambridge University Press.

Billig, M. (1991). Ideology and Opinions. Studies in Rhetorical Psychology. Londres: Sage.

Fiske, S.T. y Dupree, C. (en prensa). Cognitive processes involved in stereotyping. En R.A. Scott y S.M. Kosslyn (Eds.), Emerging Trends in the Social and Behavioral Sciences. New York: Wiley.

Íñiguez, L. (2003). El análisis del discurso en las ciencias sociales: verdades, tradiciones y práctica. En L. Íñiguez (Ed.), Análisis del discurso. Manual para las ciencias sociales (pp. 83-123). Barcelona: voc.

Kornadt, A.E. y Rothermund, K. (2011). Contexts of Aging: Assessing Evaluative Age Stereotypes in Different Life Domains.

The Journals of Gerontology: Series B: Psychological Sciences and Social Sciences, 66B(5), 547-556.

McKinlay, A. y McVittie, C. (2008). Social Psychology and Discourse. Oxford: Wiley-Blackwell.

Potter, J. y Wetherell, M. (1987). Discourse and Social Psychology: Beyond Attitudes and Behaviour. Londres: Sage. 